\title{
Coherent Diffraction Imaging
}

\author{
Jianwei Miao
}

Department of Physics \& Astronomy and California NanoSystems Institute, University of California, Los Angeles, CA 90095, USA.

For centuries, lens-based microscopy, such as light, phase-contrast, fluorescence, confocal and electron microscopy, has played an important role in the evolution of modern science and technology. In 1999, a novel form of microscopy, known as coherent diffraction imaging (CDI) or lensless imaging, was developed and transformed our traditional view of microscopy, in which the diffraction pattern of a noncrystalline object or a nanocrystal was first measured and then directly phased to obtain an image (1). The well-known phase problem is solved by combining the oversampling method with iterative algorithms. The origin of the oversampling concept can be traced back to the Shannon sampling theorem (2). When the diffraction pattern of a finite object is sampled at a frequency sufficiently finer than the Nyquist interval, the phase information is in principle encoded in the diffraction intensities (3) and can be directly retrieved by an iterative algorithm (4). Over the past decade, various CDI methods, such as plane wave CDI, Bragg CDI and ptychographic CDI, have been implemented using synchrotron radiation, X-ray free electron lasers (XFELs), high harmonic generation, optical lasers, and electrons (517).

In this talk, I will present the principle of CDI and show our recent CDI results with synchrotron radiation and XFELs. First, I will report quantitative 3D coherent x-ray diffraction imaging of a molten Fe-rich alloy and crystalline olivine sample, synthesized at $6 \mathrm{GPa}$ and $1800^{\circ} \mathrm{C}$, with nanoscale resolution (18). The 3D mass density map is determined and the 3D distribution of the Fe-rich and Fe-S phases in the olivine-Fe-S sample is observed (Fig. 1). Our results indicate that the Fe-rich melt exhibits varied 3D shapes and sizes in the olivine matrix. Second, I will present quantitative 3D coherent X-ray diffraction imaging of a whole, unstained cell at a resolution of 50-60 nm (19). We identify the 3D morphology and structure of cellular organelles including cell wall, vacuole, endoplasmic reticulum, mitochondria, granules, nucleus, and nucleolus inside the cell (Fig. 2). Finally, I will report the first experimental demonstration of single-shot 3D structure determination of an object - in this case individual gold nanocrystals at $\sim 5.5 \mathrm{~nm}$ resolution using $\sim 10$ femtosecond XFEL pulses $(20,21)$.

\section{References:}

[1]J. Miao, P. Charalambous, J. Kirz, J. and D. Sayre, Nature 400 (1999), p. 342.

[2] D. Sayre, Acta Crystallogr. A 5 (1952), p. 843.

[3] J. Miao, D. Sayre and H. N. Chapman, J. Opt. Soc. Am. A 15 (1998), p. 1662.

[4] J. R. Fienup, Appl. Opt. 21 (1982), p. 2758.

[5] I. K. Robinson et al., Phys. Rev. Lett. 87 (2001), 195505.

[6] J. Miao et al., Phys. Rev. Lett. 89 (2002), p. 088303.

[7] D. Shapiro et al., Proc. Natl. Acad. Sci. USA 102 (2005), p. 15343.

[8] M. A. Pfeifer et al., Nature 442 (2006), p. 63.

[9] J. M. Rodenburg et al., Phys. Rev. Lett. 98 (2007), p. 034801.

[10] P. Thibault et al., Science 321 (2008), p. 379.

[11] H. N. Chapman et al., Nature Phys. 2 (2006), p. 839. 
[12] M. M. Seibert et al., Nature 470 (2011), p. 78.

[13] R. L. Sandberg et al., Phys. Rev. Lett. 99 (2007), p. 098103.

[14] M. D. Seaberg et al., Opt. Express 19 (2011), p. 22470.

[15] A. Szameit et al., Nature Mater. 11 (2012), p. 455.

[16] J. Bertolotti et al., Nature 491 (2012), p. 232.

[17] J. M. Zuo et al., Science 300 (2003), p. 1419.

[18] H. Jiang et al., Phys. Rev. Lett. 110 (2013), p. 205501.

[19] H. Jiang et al., Proc. Natl. Acad. Sci. U.S.A. 107 (2010), p. 11234.

[20] K. S. Raines et al., Nature 463 (2010), p. 214-217.

[21] R. Xu et al., arXiv:1310.8594 (2013).

[22] This work is partially supported by the DARPA PULSE program through a grant from AMRDEC.
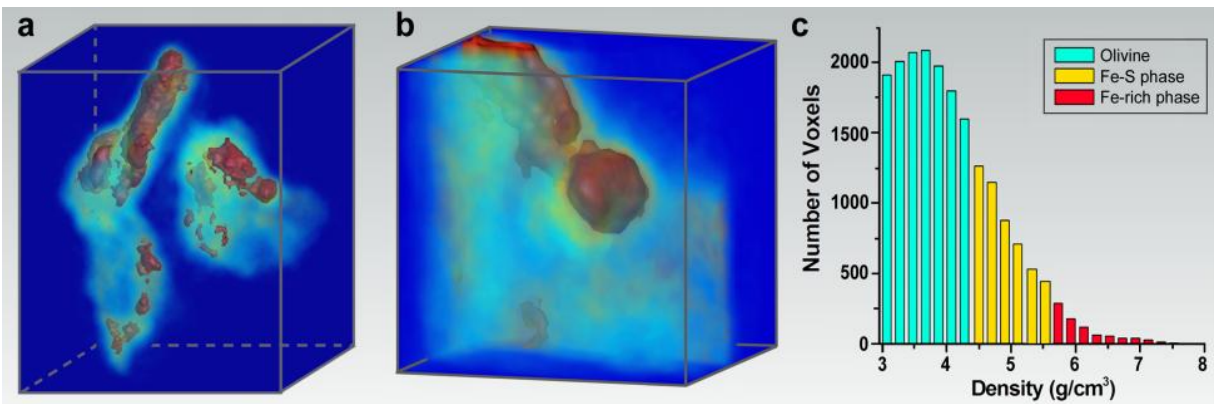

Figure 1. 3D coherent $X$-ray diffraction imaging of molten iron in mantle olivine at nanoscale resolution. (a) $3 \mathrm{D}$ volume rendering of the olivine-Fe-S sample, showing the Fe-rich phase, Fe-S phase, and olivine distribution. (b) A zoomed view of a $600 \times 600 \times 600 \mathrm{~nm}^{3}$ volume inside the olivine-Fe-S sample. (c) A histogram of the Fe-rich phase, Fe-S phase and olivine distribution in (b). (Ref. 18)

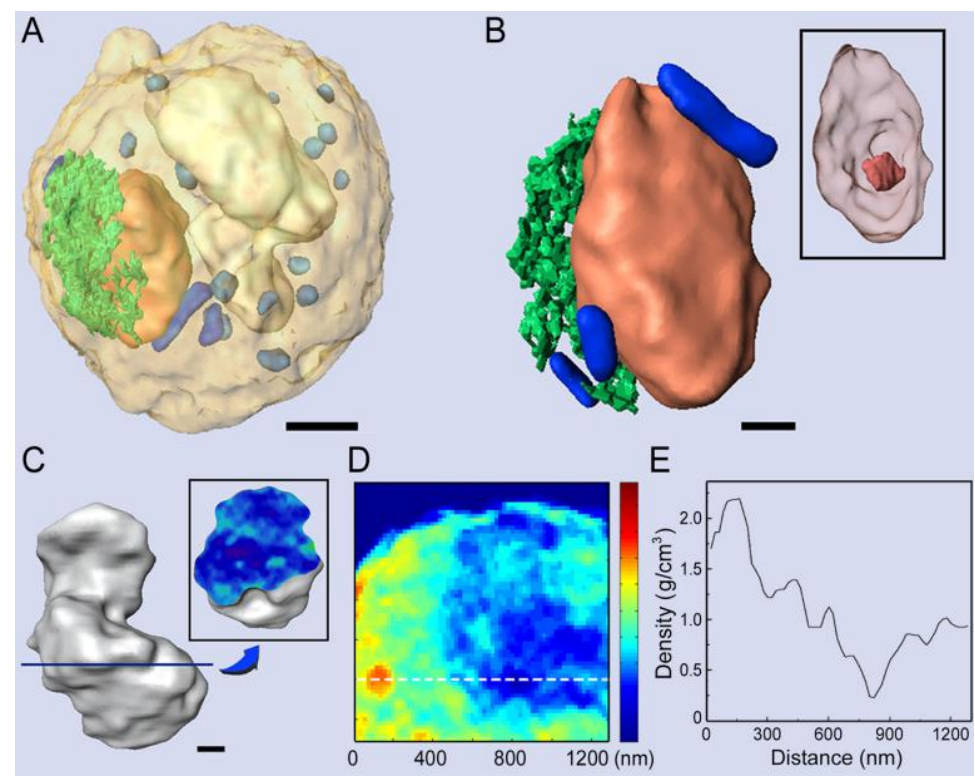

Figure 2. 3D visualization of the cellular organelles inside the yeast spore cell. (A) A 3D volume rendering of the reconstructed yeast spore, showing nucleus (orange), ER (green), vacuole (white), mitochondria (blue), and granules (light blue). (Scale bar: $500 \mathrm{~nm}$.) (B) Zoomed view of the 3D morphology and structure of the nucleus, ER, and mitochondria. Inset shows the nucleolus (orange). (Scale bar: $200 \mathrm{~nm}$.) (C) A 3D morphology and structure of the vacuole. Inset shows a cross-sectional image of the vacuole. (Scale bar: 200 nm.) (D and E) A thin slice of the reconstructed yeast spore and a line scan along the dashed line, showing the density variation across a mitochondrion and the vacuole.). (Ref. 19) 